\title{
A Signature Identification Method Based on Strength and Strokes Direction
}

\author{
Hailong Huang ${ }^{1,}$, Hong Wang ${ }^{1, b}, \mathrm{Li} \mathrm{Ji}^{1, \mathrm{c}}$ \\ ${ }^{1}$ School of Mechanical Engineering and Automation, Northeastern University, Shenyang, China \\ ahuanghailonghhl@126.com, bhongwang@mail.neu.edu.cn, ${ }^{c}$ jilirory0211@gmail.com
}

\begin{abstract}
Keywords: off-line signature identification, preprocess, mathematical morphology, structure element, real-time
\end{abstract}

\begin{abstract}
It was much more complex and difficult for off-line signature identification attributable to the limitation of available information. To solve the problem, a signature identification method based on strength and strokes direction was proposed. The signature image acquired was gray-scaled and filtered at the stage of preprocess; then the image was two-valued with different threshold based on strength feature, the regions which grayscale was less than threshold were retained; the strokes which possess distinctive directional feature were extracted by using mathematical morphology and combining different scales/directions structure element based on strokes direction feature; at last judgement was maked for sample in accordance with corresponding feature. Experimental results showed the proposed method can enhance accurate rate effectively, improve real-time performance, which was a try beneficial to apply new methods for off-line signature identification.
\end{abstract}

\section{Introduction}

The signature was applied widely in the political, economical, cultural event of society as an important identification, The criminal cases which lawbreakers carry on swindle by using fake signature were common, having brought huge economic loss and detrimental effect for society and individual. Signature has become an effective method for identification because of its uniqueness, stability and non-invasion of acquisition [1]. Signature identification was a science and technology to judge the identity of signers by analyzing and comparing the feature of signature [2]. Signature identification was divided into on-line signature identification and off-line signature identification [3]. Signature was acquired with electronic writing tablet for on-line signature identification, dynamic information such as speed, strength, signature sequence were provided; Signature was acquired with digital camera and scanner for off-line signature identification, it was much more different for off-line signature identification than on-line signature identification, because information available was fewer. Off-line signature identification becomes a "hot spot" in the area $[4,5]$, attention was concentrated on off-line signature identification in the paper.

This paper proposed a new signature identification means based on strength and strokes direction, which consists of three steps: preprocess, feature extraction and judgement. The signature image acquired was gray-scaled and filtered at the stage of preprocess. The image was two-valued with different threshold based on strength feature, the regions were retained whose grayscales were less than threshold; Strokes which possess distinctive directional feature were extracted by using mathematical morphology and combining different scales/directions structure element based on strokes direction feature at the stage of feature extraction; Judgement were maked for samples in accordance with corresponding feature at last. Experimental results showed the proposed method 
can enhance accurate rate effectively, improve real-time performance, which was a try beneficial to apply new methods for off-line signature identification, having the certain promotion application value.

\section{Preprocess of signature image}

Signature was acquired with scanner into computer in the paper, the type of image was color image, the format of image was BMP, the original signature image was showed in Figure 1 (a).

The color image needs to be gray-scaled in order to reduce the interference of unnecessary information for signature identification. The gray-scaled image mixed with a lot of noise because of the roughness of paper and ink leaved behind, the noise may severely interfere with signature identification, the paper adopted the combination median filter with paste/ delete label to filter noise. Firstly we make median filtering with $3 \times 3$ template, $3 \times 3$ template can filter the noise whose area was small, can't filter the noise whose area was big. The strokes will change if bigger template was used, we adopted paste/delete label means to filter noise whose area was big. The process of paste label was to mark the different connecting object of the image; The process of delete label was to remove connecting object which area was smaller than threshold that was configured according to the difference of area[6, 7]. The combination median filter with paste/delete label can filter noise effectively on the premise of integrity of signature, the denoised signature image was showed in Figure 1 (b) .

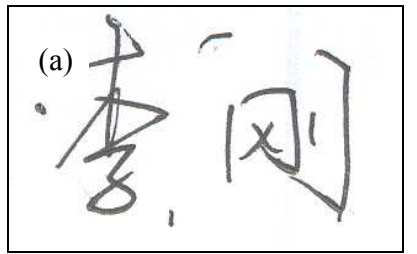

(a)-original signature image;

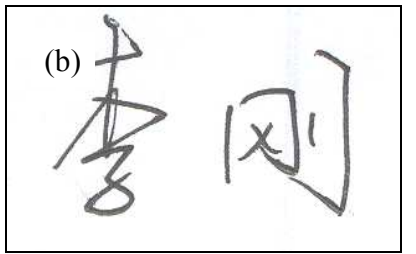

(b)-denoised signature image.

Fig. 1. Preprocess of signature image

\section{Feature extraction of signature strength based on threshold process}

Different gray signature image was produced after original signature image was gray-scaled, the difference of gray indicate the difference of signature strength: the strength was strong where the gray was small; the strength was weak where the gray was big. The regions whose grayscales were less than threshold were reserved by using threshold process, the smaller the threshold, the less the regions reserved. $0.5,0.45$ and 0.4 were chose as threshold, the signature images two-valued were showed in Figure 2. We counted the number of pixel in the regions reserved after threshold process, calculated the ratio of pixel reserved and all pixel, the ratios were shown in Table 1, we employed ten general signature as sample.
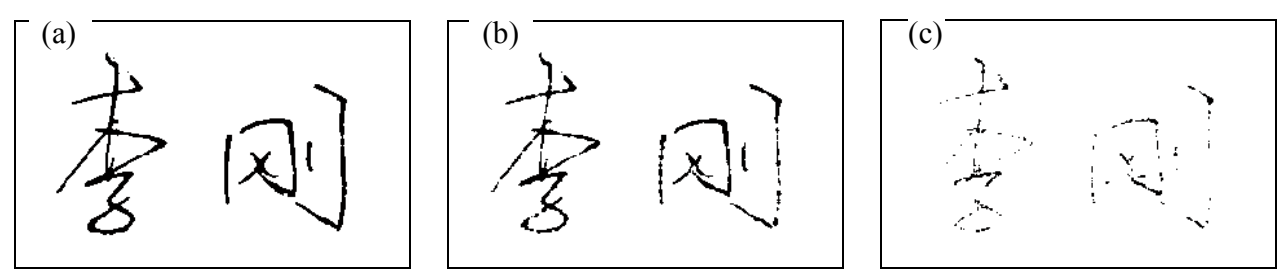

(a)-threshold $=0.5 ; \quad$ (b)-threshold $=0.45 ; \quad$ (c) -threshold $=0.4$.

Fig. 2. Threshold process of signature image 
Table 1 Ratio of pixels retained and all pixels for threshold process

\begin{tabular}{ccccccccccc}
\hline Ratio & Sample & Sample & Sample & Sample & Sample & Sample & Sample & Sample & Sample & Sample \\
& 1 & 2 & 3 & 4 & 5 & 6 & 7 & 8 & 9 & 10 \\
\hline R1 & 0.1847 & 0.1614 & 0.0711 & 0.0941 & 0.1302 & 0.1118 & 0.0890 & 0.0721 & 0.0972 & 0.0570 \\
R2 & 0.6104 & 0.5912 & 0.5084 & 0.5132 & 0.5649 & 0.5586 & 0.5494 & 0.4878 & 0.5274 & 0.4584 \\
R3 & 0.8170 & 0.8135 & 0.8111 & 0.8028 & 0.8139 & 0.8014 & 0.8126 & 0.7911 & 0.8067 & 0.7943 \\
\hline
\end{tabular}

\section{Feature extraction of strokes direction based on mathematical morphology}

The basic operations of mathematical morphology. Mathematical morphology was a new subject based on set theory, the language used to describe the mathematical morphology was set theory. Mathematical morphology was very suitable for analysis and description of signal geometrical feature, the basic concept was to probe signal using structure element, to preserve the main shape and to delete the irrelevant shape (such as noise and glitch). The structure element as a probe brings knowledge directly, such as direction, size, colour and other information, explores and researches structure features containing main information, the different structure elements produce different results $[8,9]$.

The basic operations of mathematical morphology were: dilation operation,erosion operation,open operation and close operation. In the paper $\mathrm{F}$ was regarded as image set, $\mathrm{S}$ was regarded as structure element.

Dilation operation is defined as:

$(f \oplus s)(m, n)=\max \left\{f(m-x, n-y)+s(x, y) \mid(m-x),(n-y) \in D_{f} ;(x, y) \in D_{s}\right\}$.

Erosion operation is defined as:

$(f \Theta s)(m, n)=\min \left\{f(m+x, n+y)-s(x, y) \mid(m+x),(n+y) \in D_{f} ;(x, y) \in D_{s}\right\}$.

Open operation is defined as:

$f \circ s=(f \Theta s) \oplus s$.

Close operation is defined as:

$f \bullet s=(f \oplus s) \Theta s$.

The effect of structure element on edge detection. Structure element was the basic ingredient of mathematical morphology, the difference of structure element determines directly the difference of geometric information which were analyzed and processed, also determines directly the difference of the amount of data used in computing. Therefore, the selection of structure element was primary and important step for edge detection based on mathematical morphology. The shape and size of structural elements affect the results of mathematical morphology operation: the horizontal structure element was sensitive to the vertical information; the vertical structure element was sensitive to the horizontal information; the smaller structure element was sensitive to the details; the larger structure element was sensitive to the contour [10].

Feature extraction. There were heng, shu, pie, na for strokes of signature, the direction of strokes were $0^{\circ}, 45^{\circ}, 90^{\circ}, 135^{\circ}$. We chose eight kinds of structure element to close-operate for signature image according to the basic operations of mathematical morphology and the effect of structure element, the direction of structure element were $0^{\circ}, 45^{\circ}, 90^{\circ}, 135^{\circ}$, the length of structure element were 5 and 10. Signature image was dilated and eroded with $2 \times 2$ structure 
element before close-operation to filter noise. In short, the process of operation base on mathematical morphology was showed in Formula 5, S1 was $2 \times 2$ quadrate structure element, S2 were linear structure element whose length were 5 and 10 , whose direction were $0^{\circ}, 45^{\circ}, 90^{\circ}$, $135^{\circ}$.

$$
I_{\text {out }}=I_{\text {in }} \oplus S_{1} \Theta S_{1} \bullet S_{2}
$$

The signature image was processed as Formula 5, the result were showed in Figure 3 and 4, the length of structure element was five in Figure 3; the length of structure element was ten in Fig. 4.

We counted the number of pixel in the regions reserved after operations of mathematical morphology, calculated the ratio of pixel reserved and all pixel, the ratios were shown in Table 2.
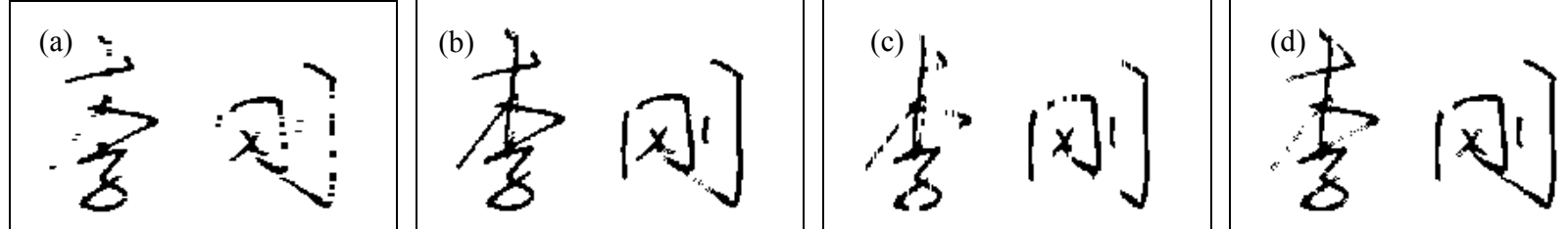

(a)-direction of $0^{\circ}$; (b)-direction of $45^{\circ}$; (c)-direction of $90^{\circ}$; (d)-direction of $135^{\circ}$

Fig. 3. Mathematical morphology operation of signature images(the length of $S=5$ )
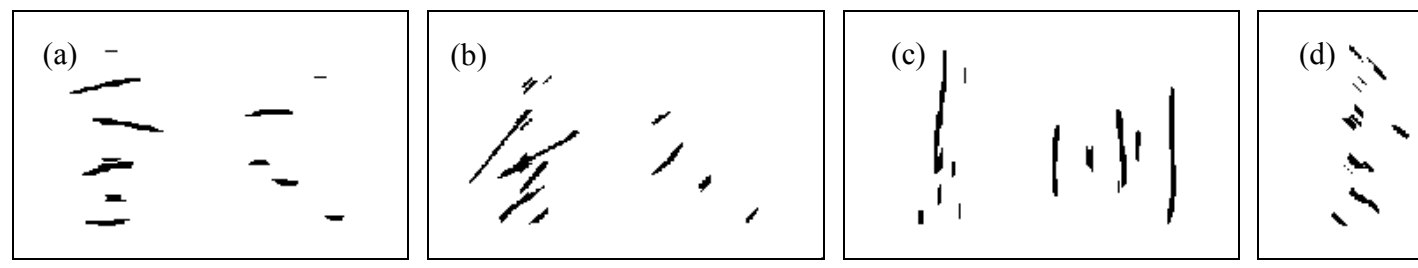

(a)-direction of $0^{\circ}$; (b)-direction of $45^{\circ}$; (c)-direction of $90^{\circ}$; (d)-direction of $135^{\circ}$

Fig. 4. Mathematical morphology operation of signature images(the length of $S=10$ )

Table 2 Rate of pixels retained and all pixels for mathematical morphology operation

\begin{tabular}{ccccccccccc}
\hline Rate & Sample & Sample & Sample & Sample & Sample & Sample & Sample & Sample & Sample & Sample \\
& 1 & 2 & 3 & 4 & 5 & 6 & 7 & 8 & 9 & 10 \\
\hline R4 & 0.7344 & 0.8376 & 0.8455 & 0.8459 & 0.7968 & 0.8086 & 0.8032 & 0.7813 & 0.7985 & 0.7706 \\
R5 & 0.9598 & 0.9735 & 0.9719 & 0.9702 & 0.9812 & 0.9683 & 0.9825 & 0.9630 & 0.9732 & 0.9791 \\
R6 & 0.8030 & 0.8492 & 0.7962 & 0.8253 & 0.8519 & 0.8488 & 0.8608 & 0.8635 & 0.8493 & 0.8317 \\
R7 & 0.9110 & 0.9433 & 0.9551 & 0.9520 & 0.9437 & 0.9317 & 0.9521 & 0.9578 & 0.9520 & 0.9523 \\
R8 & 0.3060 & 0.3045 & 0.3227 & 0.2787 & 0.3391 & 0.3250 & 0.3047 & 0.3112 & 0.3339 & 0.2958 \\
R9 & 0.3365 & 0.3772 & 0.3749 & 0.3693 & 0.3771 & 0.3856 & 0.3656 & 0.4232 & 0.3896 & 0.3526 \\
R10 & 0.4286 & 0.4534 & 0.4601 & 0.4560 & 0.4553 & 0.4539 & 0.4591 & 0.4599 & 0.4518 & 0.4677 \\
R11 & 0.3137 & 0.3183 & 0.2545 & 0.3435 & 0.3154 & 0.3396 & 0.3210 & 0.2901 & 0.2738 & 0.2867 \\
\hline
\end{tabular}

\section{Identification results and analysis}

In the paper $\mathrm{R} 1 \sim \mathrm{R} 11$ were employed to discriminate sample as identification feature, if nine in eleven features of sample belong to [ $\mathrm{min}, \max$ ], sample was considered as general signature; if two and more in eleven features of sample don't belong to [min, max], sample was considered as fake signature. Min and max of R1 R11 were showed in Table 3. 
Comparison of precision between method proposed in the paper and existing method was showed was presented, the precision of method proposed in the paper was obviously better than method based on length-width ratio and strength, slightly better than method used Gabor filter; the real-time performance of method proposed in the paper was superior to method used Gabor filter because mathematical morphology was employed

Table 3 Min and max of R1 R11

\begin{tabular}{ccccccccccccc}
\hline parameter & $\mathrm{R} 1$ & $\mathrm{R} 2$ & $\mathrm{R} 3$ & $\mathrm{R} 4$ & $\mathrm{R} 5$ & $\mathrm{R} 6$ & $\mathrm{R} 7$ & $\mathrm{R} 8$ & $\mathrm{R} 9$ & $\mathrm{R} 10$ & $\mathrm{R} 11$ \\
\hline $\min$ & 0.0570 & 0.4584 & 0.7911 & 0.7344 & 0.9598 & 0.7962 & 0.9110 & 0.2787 & 0.3365 & 0.4286 & 0.2738 \\
$\max$ & 0.1847 & 0.6104 & 0.8170 & 0.8459 & 0.9825 & 0.8635 & 0.9578 & 0.3391 & 0.4232 & 0.4677 & 0.3435 \\
\hline
\end{tabular}

\section{Conclusions}

Theoretically, increasing the number of sample will improve the precision of identification, but the number of sample was selected as ten allow for the limitation of genuine sample. If the conditions permit, the number of sample may be increased to improve the precision of identification.

The eleven feature were employed to discriminate sample, if demand all the eleven feature belong to [min, max], that was to say, increasing the standard of identification will cause the problem that discriminate general sample as fake signature. If nine in eleven features of sample belong to [min, max] , sample was considered as general signature according to the diversity of same signatory.

The method proposed in the paper has better precision for signature whose strokes were regular, the precision will decrease for signature whose strokes were irregular. Therefore we should improve the method proposed in the paper to increase generality of the method.

\section{References}

[1] Li Xin, Ding Xiao-qing. Writer identification based on improved microstructure features [D]. $J$ Tsing hua Univ (Sci \& Tech), 2010, 50(4): 595-600

[2] Plamondon R, Lorette G. Automatic signature verification and writer identification-The state of the art[J]. Pattern Recognition, 1989, 22(2): 107-131

[3] Said H E S, TAN Tieniu, Baker K D. Personal identification based on handwriting[J]. Pattern Recognition, 2000, 33(1): 149-160

[4] Bulacu M, Schomaker L. Text independent writer identification and verification using textural and allographic features[J]. IEEE Trans Pattern Analysis and Machine Intelligence, 2007, 29(2): 701-717

[5] Wang Hong, Zhao Hai-bin. Digital image processing -JAVA language implementation [M]. Shenyang: Northeastern University Press, 2005: 158-166

[6] Jing Xiao-jun. Image processing technology and application [M]. Beijing: National Defense Industry Press, 2005: 211-216

[7] Wang Jia-wen. Matlab7.6 graphics image processing[M]. Beijing: National Defense Industry Press, 2009: 264-291

[8] Lee C K, Wong S P. A mathematical morphological approach for segmenting heavily noise-corrupted images [J]. Pattern Recognition, 1996, 29(8): 1347-1358

[9] Naegel B. Using mathematical morphology for the anatomical labeling of vertebrae from 3D CT-scan images[J]. Computerized Medical Imaging and Graphics, 2007, 3: 141-156

[10] Maria C M. Fuzzy mathematical morphology: concepts and applications [J]. Vistas in Astronomy, 1996, 40(4): 469-47 